\title{
Stability of Sequences Generated by Nonlinear Differential Systems
}

\author{
By R. Leonard Brown*
}

\begin{abstract}
A local stability analysis is given for both the analytic and numerical solutions of the initial value problem for a system of ordinary differential equations. The standard linear stability analysis is reviewed, then it is shown that, using a proper choice of Liapunov function, a connected region of stable initial values of both the analytic solution and of the one-leg $k$-step numerical solution can be approximated computationally. Correspondence between the one-leg $k$-step solution and its associated linear $k$-step solution is shown, and two examples are given.
\end{abstract}

1. Introduction. The numerical solution of the initial value problem for a system of ordinary differential equations

$$
y^{\prime}(t)=f(y(t), t),
$$

and

$$
y\left(t_{0}\right)=y_{0}
$$

can be considered locally as the computation of a sequence of approximations $Y_{n}=$ $\left\{y_{n-k+1}, \ldots, y_{n-1}, y_{n}\right\}$ with each $y_{n}$ being a numerical approximation to $y\left(t_{n}\right)$ for $t_{n}=t_{0}+\sum_{i=1}^{n} h_{i}$. While many sophisticated packages [5], [7], [8], [10] exist which change the stepsize $h_{i}$ in order to achieve stability and specified accuracy, the stepsize usually remains constant for a number of steps and the change of stepsize is usually accompanied by an interpolatory change in the solution sequence $Y_{n}$, although not always [12]. For these reasons the local analysis, limited to $k$ steps, given in the sequel will assume constant stepsize $h$. The actual numerical solution element $y_{n}$ is usually computed from elements of the sequence $Y_{n-1}$ and $f(y, t)$ using one of several formulas, each of which has a local discretization error term $\phi_{n} C h^{r+1}$, where $\phi$ depends on $f$ and $t_{n}, C$ is a constant dependent on the formula, and $r$ is called the order of the method. This work is concerned with how errors are propagated when numerical methods are applied to a differential function $f(y, t)$ which is nonlinear in $y$, with emphasis on stable propagation of the errors.

This work will deal with first derivative multipstep methods of the general form

Received May 2, 1978.

AMS (MOS) subject classifications (1970). Primary 34D20, 65 L05.

Key words and phrases. Initial value problem, numerical integration of ordinary differential equations, discrete Liapunov function, stability of nonlinear sequences.

*This work was supported in part by NASA grant NSG 1335. 


$$
y_{n}=\sum_{i=1}^{k} \alpha_{i} y_{n-i}+h \sum_{i=0}^{k} \beta_{i} f\left(y_{n-i}, t_{n-i}\right),
$$

which have a diversity of specific forms depending on the intended applications. For example, the Adams-Bashforth and Adams-Moulton formulas are of the form

$$
\begin{aligned}
& y_{n}=y_{n-1}+h \sum_{i=1}^{k} b_{i} f\left(y_{n-i}, t_{n-i}\right), \\
& y_{n}=y_{n-1}+h \sum_{i=0}^{k} b_{i}^{*} f\left(y_{n-i}, t_{n-i}\right),
\end{aligned}
$$

respectively. A $k$-step Adams-Bashforth formula is of order $k$, and a $k$-step AdamsMoulton of order $k+1$. Many methods iterate (4) a set number of times, often one, rather than to convergence to an iterative solution.

The concept of multistep methods is useful since order of accuracy as high as $k+1$ [3] can be achieved by a stable formula with only a few evaluations of $f(y, t)$, whereas a correspondingly accurate Runge-Kutta formula [2] requires at least one evaluation of $f(y, t)$ for each additional order of accuracy. Newer methods have been developed to handle special circumstances. The backward differentiation implicit formulas

$$
y_{n}=\sum_{i=1}^{k} a_{i} y_{n-i}+h b_{0} f\left(y_{n}, t_{n}\right)
$$

are useful in solving stiff systems of equations [5].

2. Stability of Solution Sequences. The standard linear stability analysis is formula specific and describes the behavior of a numerical formula applied to the complex test equation $y^{\prime}=\lambda y, y\left(t_{0}\right) \neq 0$. Let $E_{n}=\left\{e\left(t_{n-k+1}\right), \ldots, e\left(t_{n-1}\right)\right.$, $\left.e\left(t_{n}\right)\right\}$ be the difference sequence for $y\left(t_{n}\right)-z\left(t_{n}\right)$, where each solves the test equation for a different initial value $y_{0}, z_{0}$. Then $e\left(t_{n}\right)=\left(y_{0}-z_{0}\right) e^{\lambda t}$ is nonincreasing in norm for $\operatorname{Re}(\lambda) \leqslant 0$. Such a condition is called stability. It is desirable for the numerical solution $y_{n}$ to be stable if the true solution is stable, so for a given stepsize $h$, one finds all complex $\lambda$ such that any numerical sequence $\hat{E}_{n}=\left\{e_{n-k+1}\right.$, $\left.\ldots, e_{n-1}, e_{n}\right\}$ has the property $\left|e_{i+1}\right| \leqslant\left|e_{i}\right|$ for all $i$, where $e_{i}=y_{i}-z_{i}$, the difference between the numerical solution sequences with initial values $y_{0}, z_{0}$.

For Euler's formula, $y_{n}=(1+h \lambda) y_{n-1}$, hence the numerical solution is stable for $|1+h \lambda| \leqslant 1$. For multistep formulas, linear stability is characterized by the generating polynomials

$$
\begin{aligned}
& \rho(\xi)=\sum_{i=0}^{k} a_{i} \xi^{k-i} \\
& \sigma(\xi)=\sum_{i=0}^{k} b_{i} \xi^{k-i}
\end{aligned}
$$

where $\rho$ and $\sigma$ have no common divisors. The region of linear stability is all $h \lambda$ such 
that $\rho(\xi)+h \lambda \sigma(\xi)$ has all roots inside the unit circle, or on the unit circle and simple [6]. For Euler's formula, $\rho(\xi)+h \lambda \sigma(\xi)$ is $(-\xi+1)+h \lambda$. Since this analysis is formula specific, to investigate the formula's effect on an actual $f(y, t)$ one considers all the eigenvalues $\lambda_{i}$ of the Jacobian matrix $\partial f(y, t) / \partial y$; if all $h \lambda_{i}$ are inside the stability region for all $y_{n}, t_{n}$ of interest, then the numerical solution will be stable. Insuring that such a condition holds is usually not desirable and often is impossible.

To develop a stability analysis for nonlinear $f(y, t)$, let $f(y, t)$ have the property

$$
\operatorname{Re}\langle y-z, f(y, t)-f(z, t)\rangle \leqslant \mu\|y-z\|^{2}
$$

for all $t, y$, and $z$ of interest. Here $\langle u, v\rangle=u * Q v$ for some positive definite Hermitian matrix $Q$, and $\|u\|^{2}=\langle u, u\rangle$. Then for any two solutions $y(t), z(t)=y(t)-e(t), e(t)$ satisfies

$$
\frac{d e(t)}{d t}=f(y(t), t)-f(z(t), t)
$$

and (8) implies that

$$
\frac{d}{d t}\|e(t)\|^{2}=2 \operatorname{Re}\left\langle e(t), \frac{d e(t)}{d t}\right\rangle \leqslant 2 \mu\|e(t)\|^{2} ;
$$

and thus

$$
\|e(t)\| \leqslant e^{\mu t}\left\|e\left(t_{0}\right)\right\|
$$

which is nonincreasing for $\mu \leqslant 0$.

However, (8) is again a condition that cannot be easily verified, so a concept relating the true solution sequence $Y_{n}^{t}=\left\{y\left(t_{n-k+1}\right), \ldots, y\left(t_{n-1}\right), y\left(t_{n}\right)\right\}$ to the computed sequence $Y_{n}^{c}=\left\{y_{n-k+1}, \ldots, y_{n-1}, y_{n}\right\}$ is presented in the sequel. The following definitions and theorem are helpful. They occur in Dahlquist [4], and the theorem proof is presented since it occurs only in a technical report.

Definition. A linear $k$-step formula satisfies

$$
0=\sum_{j=0}^{k}\left[a_{j} \hat{y}_{n-j}+h b_{j} f\left(\hat{y}_{n-j}, t_{n-j}\right)\right] .
$$

Definition. A one-leg $k$-step formula corresponding to (9) satisfies

$$
0=\sum_{j=0}^{k} a_{j} y_{n-j}+h s f\left(\frac{1}{s} \sum_{j=0}^{k} b_{j} y_{n-j}, \frac{1}{s} \sum_{j=0}^{k} b_{j} t_{n-j}\right),
$$

where $s=\sigma(1)$. Without loss of generality, set $s=1$.

THEOREM 1 [4]. Let $Y_{n}$ be a sequence which satisfies (10), and let $\hat{Y}_{n}=$ $\left\{\hat{y}_{n}\right\}$ be such that

$$
\hat{y}_{n}=\sum_{j=0}^{k} b_{j} y_{n-j}=\sigma(E) y_{n}
$$

where $E$ denotes the back shifting operator. Then $\hat{Y}_{n}$ satisfies (9). Conversely, if $\hat{Y}_{n}$ satisfies (9), then there exists a sequence $Y_{n}$ such that $\hat{y}_{n}=\sigma(E) y_{n}$, and $y_{n}$ satisfies (10). 
Proof. Without loss of generality assume the system of equations is autonomous. Write $(10)$ as $\rho(E) y_{n}=-h f\left(\sigma(E) y_{n}\right), n=0,1,2, \ldots$ This together with (11) implies $\rho(E) \hat{y}_{n}=\rho(E) \sigma(E) y_{n}=-h \sigma(E) f\left(\hat{y}_{n}\right)$, which implies that $\hat{y}_{n}$ satisfies (9).

For the converse, Euclid's theorem on polynomials with no common divisors implies the existence of polynomials $P, Q$ which satisfy $P(x) \sigma(x)+Q(x) \rho(x)=x^{m}$, $0 \leqslant m \leqslant k$. Writing (9) as $\rho(E) \hat{y}_{n}=-h \sigma(E) f\left(\hat{y}_{n}\right)$ and setting $y_{n}=$ $E^{-m}\left(P(E) \hat{y}_{n}-h Q(E) f\left(\hat{y}_{n}\right)\right)$ gives

$$
\sigma(E) y_{n}=E^{-m}\left(P(E) \sigma(E) \hat{y}_{n}+Q(E) \rho(E) f\left(\hat{y}_{n}\right)\right)=E^{-m}(P(E) \sigma(E)+Q(E) \rho(E)) \hat{y}_{n},
$$

which gives $\sigma(E) y_{n}=\hat{y}_{n}$. Next, set

$\rho(E) y_{n}=-E^{-m} h(P(E) \sigma(E)+Q(E) \rho(E)) f\left(\hat{y}_{n}\right)=-h f\left(\hat{y}_{n}\right)=-h f\left(\sigma(E) y_{n}\right), \quad n \geqslant m$,

and $Y_{n}$ satisfies (10), proving the converse.

This shows that $Y_{n}$ given by the one-leg $k$-step formula will have similar stability properties to its corresponding linear $k$-step sequence $\hat{y}$. Dahlquist [4] has described a discrete Liapunov function $V_{G, l, h}$ which, applied to a sequence $Y_{n}$, characterizes the stability of that sequence generated by a nonlinear system (1). Let $V_{G, l, h}\left(Y_{n}\right)=\Sigma_{i=l}^{l} \Sigma_{j=1}^{l} g_{i j}\left\langle y_{n-i+1}, y_{n-j+1}\right\rangle$, where $G$ is a positive definite, $l \times l$ matrix. The structure of $G$ assures that $V_{G, l, h}$ is positive for $Y_{n} \neq\{0\}$.

Definition. The $(G, l, h)$-domain of attraction of (the numerical solution to) the system (1) is all $z_{0}$ such that $\Delta V_{G, l, h}\left(Z_{0}\right)=V_{G, l, h}\left(Z_{1}\right)-V_{G, l, h}\left(Z_{0}\right) \leqslant 0$, where $Z_{0}=\left\{z((1-l) h), \ldots, z(-h), z_{0}\right\}, Z_{1}=\left\{z((2-l) h), \ldots, z_{0}, z_{1}\right\}$ for the numerical solution and $Z_{1}=\left\{z((2-l) h), \ldots, z_{0}, z(h)\right\}$ for the exact solution.

Definition. The $(G, l, h)$-stability region of (the numerical solution to) the nonlinear system (1) is all $z_{0}$ such that

$$
V_{G, l, h}\left(Z_{0}\right) \leqslant \inf _{z_{0} \in \partial D}\left\{V_{G, l, h}\left(Z_{0}\right)\right\},
$$

where $\partial D$ is the boundary of the $(G, l, h)$-domain of attraction.

This has the following application. Rather than requiring that $\operatorname{Re}\langle y-z, f(t, y)-f(t, z)\rangle \leqslant 0$, a connected subset of initial values $y_{0}$ is found such that $y(h)$ will be in that subset if $y_{0}$ was; this is the stability region. This insures that the difference $y(h)-z(h)$ is bounded since both $y(h)$ and $z(h)$ are in the stability region if $y_{0}$ and $z_{0}$ were. If $f(y, t)$ is autonomous, $y\left(t_{n}\right)$ will remain in the region as $n \rightarrow \infty$. For most well-behaved functions $f(y, t)$, the boundary of the region around a stable point can be approximated computationally. Once the analytic stability region is known, the numerical stability region can be calculated using the one-leg $k$-step method for the same sequence $\left\{y((1-k) h), \ldots, y(-h), y_{0}\right\}$ to get $y_{1}$. The two regions can then be compared.

Analytically, it is possible to form a particular $G$, based on the coefficients of a one-leg $k$-step method, such that all numerical sequences based on $f(t, y)$ that satisfy (8) will have a stable solution. Liniger and Odeh [9] have shown how to pick $G$ for second order two-step formulas, second order three-step formulas, and third order three-step formulas.

It is shown below that even an arbitrary choice of the positive definite Hermitian 
matrix $G$ will generate some usable results, and Theorem 2 demonstrates that using some $G$ for a one-leg $k$-step solution, $y_{n}$ will generate the same stability region for the related solution $\hat{y}_{n}$ of the linear $k$-step formula for a modified $\hat{G}$.

THEOREM 2. If $V_{G, l, h}\left(Y_{n}\right)=c$ for the symmetric positive definite matrix $G$, then there exists a symmetric, positive definite matrix $\hat{G}$, dependent only on $G$ and $\sigma(x)$, such that $V_{\hat{G}, l, h}\left(\hat{Y}_{n}\right)=c$, where $\hat{y}_{n}=\sigma(E) y_{n}$ are the elements of $\hat{Y}_{n}$ and $Y_{n}$.

Proof. Without loss of generality, consider a system of only one equation $y^{\prime}=$ $f(y, t)$ generating the sequence $Y_{n}=\left\{y_{n-k+1}, \ldots, y_{n}\right\}$. Since $\hat{y}_{n}$ and $y_{n}$ are related only by the $k+1$ coefficients of $\sigma(x)$, replace the sequences by the vectors $w_{n}=\left(y_{n}, y_{n-1}, \ldots, y_{n-k+1}\right)^{*}$ and $w_{n}^{\prime}=\left(y_{n}, y_{n-1}, \ldots, y_{n-l-k+1}\right)^{*}$, where $*$ is the transpose operator. Let $G^{\prime}$ be a $(k+l)$ by $(k+l)$ matrix consisting of $G$ in the upper $l$ by $l$ partition, and 0 elsewhere. Then $V\left(w_{n}\right)=w_{n}^{*} G w_{n}=c>0$, and $V\left(w_{n}^{\prime}\right)=w_{n}^{\prime *} G^{\prime} w_{n}^{\prime}=c$.

Define $S$ such that $\hat{w}_{n}=S w_{n}^{\prime}$, thus

$$
S=\left(\begin{array}{ccccccc}
b_{0} & b_{1} & \cdots & b_{k} & 0 & \cdots & 0 \\
0 & b_{0} & & b_{k-1} & b_{k} & \cdots & 0 \\
& & & \cdot & & & \\
\vdots & & & & \cdot & & \\
0 & & & b_{0} & b_{1} & \cdots & b_{k}
\end{array}\right)
$$

is an $l$ by $l+k$ matrix. Then since $G^{\prime}$ is of rank $l$ because $G$ is positive definite, there exists a singular value decomposition of $G^{\prime}=U F V^{*}$ for $U, V l+k$ by $l+k$ unitary matrices, and $F=\left(\begin{array}{ll}D & 0 \\ 0 & 0\end{array}\right)$ for $D$ an $l$ by $l$ diagonal matrix of singular values of $G$. Thus, there exists an $l$ by $l$ matrix $\hat{G}$ such that $G^{\prime}=S^{*} \hat{G} S$. This is seen by letting $S$ have the singular value decomposition $U_{s}(\Sigma \mid 0) V_{s}$, where $\Sigma$ is an $l$ by $l$ diagonal matrix. Then

$$
\hat{G}=U_{s}\left(\Sigma^{-1} \mid 0\right) V_{s}^{*} U F V^{*} V_{s}^{*}\left(\Sigma^{-1} \mid 0\right)^{*} U_{s}^{*} \text {. }
$$

If $b_{0}, b_{1}, \ldots, b_{i-1}$ are all zero, a similar argument can be made using an $l$ by $l+k-i$ matrix $S$ where $b_{i}$ is the coefficient of lowest index $i$ such that $b_{i} \neq 0$, and

$$
S=\left(\begin{array}{cccccc}
b_{i} & b_{i+1} & \cdots & b_{k} & \cdots & 0 \\
0 & b_{i} & \cdots & b_{k-1} & \cdots & 0 \\
0 & \cdots & \cdots & & & 0
\end{array}\right) .
$$

Thus, there exists a $\hat{G}$ dependent only on $\sigma(x)$ such that $V_{\hat{G}, l, h}\left(Y_{n}\right)=c$ for all $c$, which was to be shown.

3. Computational Experience. Preliminary computational results have indicated the usefulness of $(G, k, h)$-stability. Two test functions have been investigated. The first is linear and was used to verify that similar results are given by both linear and 
nonlinear analyses of a linear equation. Let $y^{\prime}=\lambda_{0} y, \lambda_{0}=\left(\ln y_{0}\right) / h$, with solution sequence $y_{n}=y_{0}^{n+1}$, be the test equation. The exact stability region, for any $k$ and any $h$, is the unit circle, corresponding to $\left|y_{0}\right| \leqslant 1$ and $\operatorname{Re}(\lambda h) \leqslant 0$.

Figure 1 shows the corresponding $(I, k, .032)$-stability region for Euler's method $(k=1)$, Euler predictor with one backward Euler corrector $y_{n}=y_{n-1}+h f\left(y_{n}, t_{n}\right)$ $(k=1)$, and second order Adams-Bashforth explicit method $(k=2)$.

It is interesting that this solution sequence solves the single first order difference equation $y_{n+1}=y_{0} y_{n}$, yet none of the sophisticated integration formulas can produce a stable solution throughout the same region, although they, too, are $k$-step difference equations. The best is Euler's method, $y_{n}=(1+h \lambda) y_{n-1}$, probably because $(1+h \lambda)=1+\ln y_{0}$ is a good approximation to $y_{0}$ away from the origin.

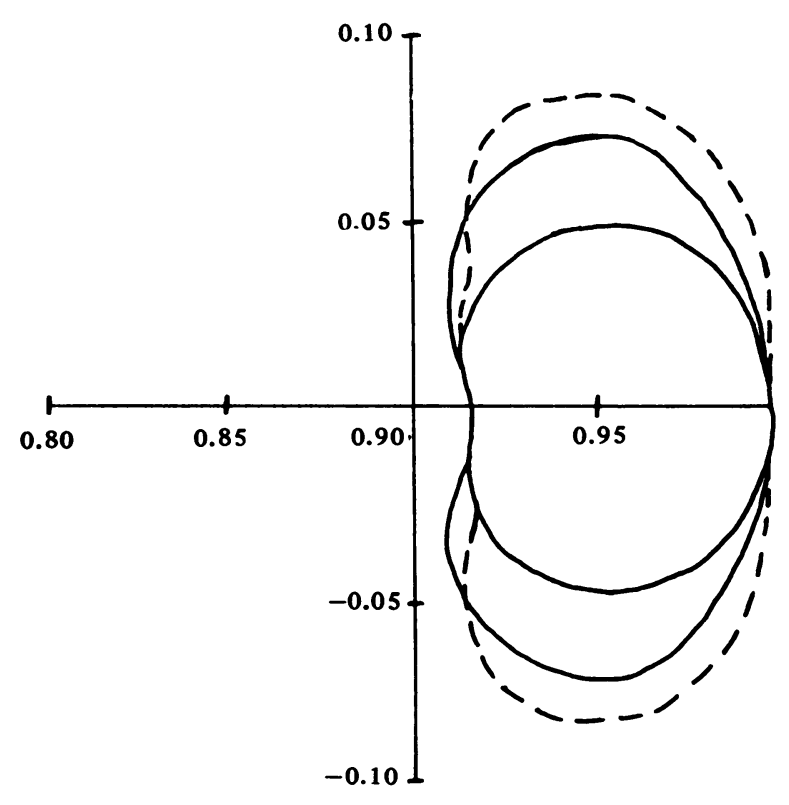

FIgURE 1. Numerical stability regions for Euler predictor (smallest area), Euler predictor with one backward Euler corrector (dashed lines), and 2 step explicit Adams formula (intermediate area).

A more practical two dimensional problem is the $(I, k, .032)$ stability of the longitudinal equations of motion of a gliding jet aircraft [1], [11]:

$$
\begin{aligned}
& \dot{u}=-g \sin \theta-w q+\left[C_{1} V^{2}+C_{2} q V\right], \\
& \dot{w}=g \cos \theta+u q+\left[C_{3} V^{2}+C_{4} q V\right], \\
& \dot{\theta}=q \\
& V=\left(u^{2}+w^{2}\right)^{1 / 2}, \\
& \dot{q}=k_{1}+k_{2} q / V .
\end{aligned}
$$




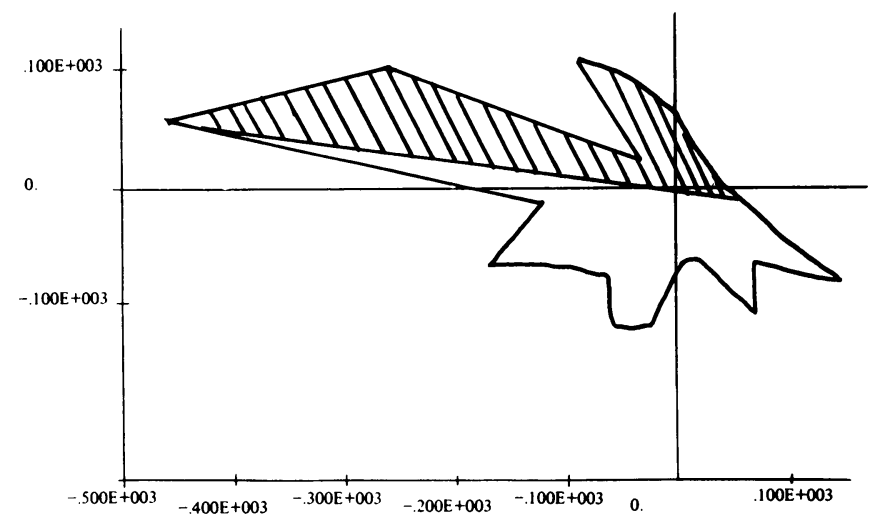

FIGURE 2. Exact stability region $(I, k, .032)$ for $k=1$ (larger), and $k=2$.

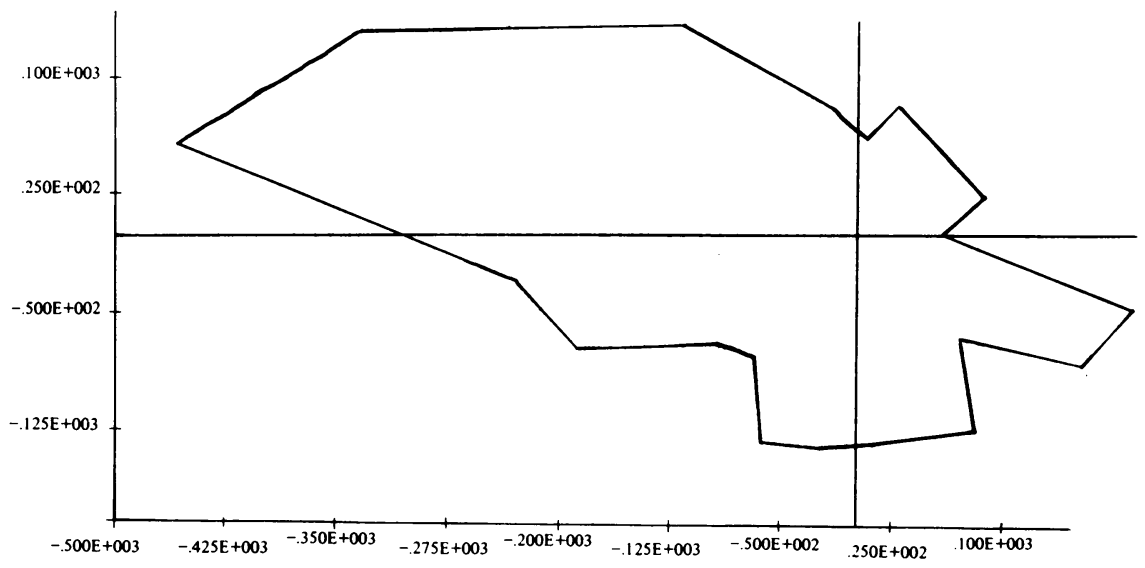

Figure 3. $(I, 1, .032)$ stability region for Euler predictor and backward Euler corrector.

The coefficients $C_{i}, k_{i}$ are determined experimentally at a constant trimmed flight state with $\theta_{0}$ given. Under the simplifying assumption that $\dot{q}=0$ ( $q$ is the rotational velocity in radians/sec), the resulting constant $q$ can be gotten from the last equation given $u_{0}$ and $w_{0}$, and thus $\theta=\theta_{0}+t q$ replaces this equation, leaving only $u$ and $w$.

By using the power series method on $u(t), w(t), V(t)$, and $V^{2}(t)$ for $|t|<1$, the exact solution for 30 steps forward or backward can be computed to machine accuracy by recursively computing as many coefficients of the power series as needed. Figure 2 shows 25 connected points of the exact $(I, 1, .032)$ and $(I, 2, .032)$ stability region about $u=600, w=0$. Figure 3 shows 25 connected points of the $(I, 1, .032)$ stability region for forward Euler predictor with a backward Euler corrector. Figure 4 is the $(I, 2, .032)$ region for the Adams-Bashforth 2-step explicit method.

For any system for which a stable initial point is available, it is possible to 


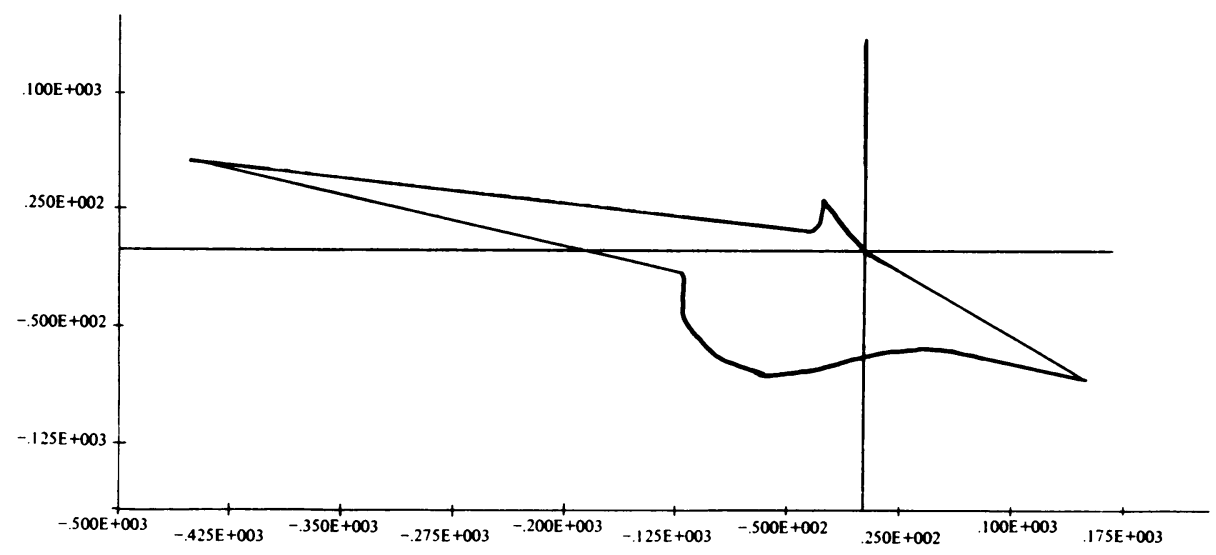

FiguRE 4. (I, 2, .032) stability region for Adams 2-step explicit method.

compute the discrete stability region of the numerical solution and graph any two coordinates. If a power series solution is also available and a discrete Liapunov stability region exists, these stability regions can be compared with the true region to determine which of the numerical methods under consideration is most appropriate, from a stability consideration, for use in solving the system numerically. This is especially useful in applications such as real time simulation for which the same set of equations is solved for every simulation run and for constant stepsize. It also allows methods specially tailored to the problem to be considered at a computer terminal session, rather than analytically. Further work should be undertaken concerning best choice of $G$, computation of the exact stability region, and existence of the exact stability region, but the software developed to date is already useful in several applications.

Department of Applied Mathematics and Computer Science University of Virginia

Charlottesville, Virginia 22903

1. R. L. BROWN, "Graphical stability comparison of analytic and numerical solutions of nonlinear systems," Numerical Methods for Differential Equations and Simultation, A. W. Bennett and R. Vichnevetsky (eds.), North-Holland, Amsterdam, 1978.

2. J. C. BUTCHER, "On the attainable order of Runge-Kutta methods," Math. Comp., v. 19, 1965, pp. 408-417.

3. G. DAHLQUIST, "Numerical integration of ordinary differential equations," Math. Scand., v. 4, 1956, pp. 33-50.

4. G. DAHLQUIST, On Stability and Error Analysis for Stiff Non-Linear Problems, Report NA-7508, Dept. of Information Processing, Royal Institute of Technology, Stockholm, 1975.

5. C. W. GEAR, "Algorithm 407: DIFSUB for solution of ordinary differential equations," Comm. $A C M$, v. 14, 1971 , pp. 185-190.

6. C. W. GEAR, Numerical Initial Value Problems in Ordinary Differential Equations, Prentice-Hall, Englewood Cliffs, N. J., 1971.

7. A. C. HINDMARSH, GEAR: Ordinary Differential Equation System Solver, UCID300001 , Rev. 2, Lawrence Livermore Lab., Livermore, California, 1972.

8. F. T. KROGH, "A variable step variable order multistep method for the numerical solution of ordinary differential equations," Information Processing 68, Vol. I, A. J. H. Morrell (ed.), North-Holland, Amsterdam, 1969, pp. 194-199. 
9. W. LINIGER \& F. ODEH, On Liapunov Stability of Stiff Non-Linear Multistep Difference Equations, AFOSR-TR-76-1023, IBM Thomas J. Watson Research Center, 1976.

10. L. F. SHAMPINE \& M. K. GORDON, Computer Solution of Ordinary Differential Equations: Initial Value Problems, Freeman, San Francisco, Calif., 1976.

11. G. G. STEINMETZ, R. V. PARRISH \& R. L. BOWLES, Longitudinal Stability and Control Derivatives of a Jet Fighter Airplane Extracted from Flight Test Data by Utilizing Maximum Likelihood Estimation, NASA-TV D-6532, NASA Langley Research Center, Hampton, Va., 1972.

12. K. W. TU, Stability and Convergence of General Multistep and Multivalue Methods with Variable Stepsize, UIUCDCS-R-72-526, Univ. of Illinois, Urbana, Ill., 1972. 